\title{
Video Article \\ Dry Powder and Nebulized Aerosol Inhalation of Pharmaceuticals Delivered to Mice Using a Nose-only Exposure System
}

\author{
Jonathan E. Phillips ${ }^{1}$, Xuxia Zhang ${ }^{1}$, James A. Johnston ${ }^{1}$ \\ ${ }^{1}$ Inflammation Research, Amgen \\ Correspondence to: Jonathan E. Phillips at Jonathan.phillips@amgen.com \\ URL: https://www.jove.com/video/55454 \\ DOI: doi:10.3791/55454
}

Keywords: Medicine, Issue 122, Inhaled therapy, pre-clinical, dust feed, jet nebulizer, ipratropium, bronchoconstriction, intratracheal

Date Published: 4/6/2017

Citation: Phillips, J.E., Zhang, X., Johnston, J.A. Dry Powder and Nebulized Aerosol Inhalation of Pharmaceuticals Delivered to Mice Using a Noseonly Exposure System. J. Vis. Exp. (122), e55454, doi:10.3791/55454 (2017).

\section{Abstract}

Obstructive respiratory diseases like asthma and chronic obstructive pulmonary disease (COPD) are currently treated by inhaled antiinflammatory and bronchodilator drugs. Despite the availability of multiple treatments, both diseases are growing public health concerns. The majority of asthma patients are well controlled on current inhaled therapies but a substantial number of patients with severe asthma are not. Asthma affects an estimated 300 million people worldwide and approximately 20 percent have a severe form of the disease. In contrast to asthma, there are few effective therapies for COPD. An estimated $10 \%$ of the population has COPD and the trend in death rates is increasing for COPD while decreasing for other major diseases. Although developing drugs for inhaled delivery is challenging, the nose-only inhalation unit enables direct delivery of novel drugs to the lung of rodents for pre-clinical efficacy and safety/toxicology studies. Inhaled drug delivery has multiple advantages for respiratory diseases, where high concentration in the lung improves efficacy and minimizes systemic side effects. Inhaled corticosteroids and bronchodilators benefit from these advantages and inhaled delivery may also hold potential for future biologic therapies. The inhalation unit described herein can generate, sample for characterization, and uniformly deposit a drug aerosol in the lungs of rodents. This enables the pre-clinical determination of the efficacy and safety of drug doses deposited in the lungs of rodents, key data required before initiating clinical development.

\section{Video Link}

The video component of this article can be found at https://www.jove.com/video/55454/

\section{Introduction}

There are many advantages to inhaled administration of drugs for the treatment of respiratory disease. Inhaled delivery applies the therapeutic agent directly to the site of action, the lungs. A high local concentration of the drug in the lungs offers a significant advantage that minimizes dose and systemic exposure, and maximizes efficacy. This is an important advantage that can greatly increase the therapeutic index (TI, ratio of dose of drug that causes a side-effect over the dose of drug that provides efficacy) of a drug. Inhaled $\beta_{2}$-adrenergic agonists, corticosteroids, and anti-cholinergic drugs have proven effective at improving lung function in patients with asthma and COPD, while minimizing the systemic side effects (tachycardia, immunosuppression, and constipation) observed when these drugs are taken orally. New drug classes (for example, PDE4 inhibitors ${ }^{1}$ and BTK inhibitors ${ }^{2}$ ) have recently proven effective at improving lung function in pre-clinical animal disease models but, similar to $\beta_{2}$-agonists, corticosteroids, and anti-cholinergic drugs, suffer from systemic side effects that may be minimized by inhaled delivery. Due to the added cost of development of inhaled vs. oral drugs, an inhaled formulation should only be considered for respiratory indications after successful oral/systemic administration reveals dose limiting mechanism-based systemic side effects.

Pre-clinically, inhaled compounds are optimized to increase the TI, which requires in vivo efficacy and side effect measurements. Initially these measurements can be made in separate assays, usually a topically delivered efficacy measurement and a systemically delivered side-effect measurement, but to truly compare compounds, efficacy and side effects should be measured in the same animals after inhaled administration. This requires dose/response studies that achieve enough compound administered to the lungs to induce a measureable side effect. The only way currently to uniformly distribute large doses of drug into the lungs of multiple small animals simultaneously is nose-only inhalation ${ }^{3,4,5}$. The strengths and weaknesses of different inhalation exposure techniques have been recently reviewed ${ }^{6,7,8}$. Specialized equipment and a large amount of test compound (gram quantities) are required for nose-only inhaled drug delivery, but proof-of-concept studies may be possible by other means.

When the amount of drug is limited (mg quantities), direct administration methods are an option but all suffer from non-homogeneous deposition, with more drug concentrated along the central airways and less well represented in the parenchymal/alveolar regions ${ }^{3,4,5}$. The effective dose delivered by direct instillation is always higher and can never be directly compared to inhaled doses ${ }^{4}$. Direct instillation methods including intranasal ${ }^{9}$, intratracheal liquid ${ }^{10,11}$ and spray instillation ${ }^{12}$, or dry-powder insufflation ${ }^{13,14}$ can be used as a screening tool to determine the approximate dose range for later nose-only inhalation studies, or to determine the ranking of efficacy/toxicity for a series of structurally similar 
drugs $^{15}$. Due to the central airway deposition pattern, direct administration methods may be more useful to determine the effects of compounds that act on the central airways (bronchodilators or mast cell inhibitors) than in the peripheral lung (anti-inflammatories).

Unlike humans, who can inhale a substantial dose of concentrated aerosol from an inhaler in a single deep breath, continuous generation of a respirable (0.5-5 $\mu \mathrm{m}$ mass median aerodynamic diameter, MMAD) aerosol, for up to an hour, is required to deposit an efficacious drug dose into a spontaneously breathing rodent's lungs in a nose-only inhalation system ${ }^{16}$. Aerosol generators (jet nebulizer or the Wright dust feed ${ }^{17}$ ) that can continuously produce the required aerosol particle size and concentration for nose-only inhalation studies are not very efficient at generating high quality (respirable) aerosols. The drug feed rates to these aerosol generators for potent (IC $\mathrm{C}_{50} \mathrm{pM}$ to $\mathrm{nM}$ in functional cell based assays) compounds are commonly in the 1 to $10 \mathrm{mg} / \mathrm{min}$ range and usually less than $1 \%$ of that drug aerosol makes it to the breathing zone of the animals (Figure 1). Many of the particles generated are too big $(>5 \mu \mathrm{m})$ to enter the lungs and are removed by an aerosol classifier (a pre-separator or cyclone with a $5 \mu \mathrm{m}$ cut point) to avoid a large dose of drug in the nose. Adding to the inefficiency of nose-only inhalation systems is the small particle size range ( 0.5 to $5 \mu \mathrm{m}$ MMAD) for respirable particles. Many of the aerosol particles less than $0.5 \mu \mathrm{m}$ are exhaled (like cigarette smoke) and not deposited in the lungs ${ }^{18}$. Also, many of the "larger" aerosol particles $(\sim 5 \mu \mathrm{m})$ deposit in the nose are absorbed or transported by mucociliary clearance towards the back of the throat where they are swallowed into the stomach ${ }^{19}$. When using nose-only inhalation, the dose deposited in the nose is always larger than the dose deposited in the lungs and the nasal dose can contribute to systemic exposure and side-effects ${ }^{20}$. Inherently, inhaled drug doses are small (in the microgram range) minimizing any systemic side effect potential of drug absorbed by the nasal, lung, or gastrointestinal tissues. Even when the particle size of the aerosol supplied to the animals is in the respirable range, an average of only $4 \%$ of the aerosol particles that make it to the breathing zone of the animals deposit in the lungs. More efficient aerosol generators are available, but the jet nebulizer and Wright dust feed are unmatched for their ability to produce continuous aerosols from diverse liquid and dry powder formulations, respectively.

Respirable aerosol from the pre-separator passes into the nose-only exposure inhalation unit ${ }^{21}$ that is based on a flow-past the nose design ${ }^{22}$. The inhalation unit has 3 tiers (only two tiers are shown in Figure 1) and each tier contains 10 exposure ports for animals and aerosol sampling. The ports are located peripherally around the central aerosol plenum. Conscious mice are placed in glass restraining holders (6 inches long by 1.2 inch diameter) and breathe the aerosol contents of the inhalation unit. The mice are not acclimated to the restraint devices ${ }^{23}$. Previous experience has shown that mice tolerate tube restraint of less than an hour duration similarly, with or without adaptation ${ }^{2}$.

The inhalation unit is designed to deliver drug aerosols directly to the lungs of the animals while avoiding exposure to the operators. The potency/toxicity of these drugs is usually unknown and multiple engineering safety controls are used to avoid exposure to the operators. The operators must always wear personal protective equipment (gloves, laboratory coats, respirators, and safety glasses). The outer plenum of the inhalation unit is under negative pressure at all times during operation, allowing for the safe removal of single or groups of animals without shutting off the aerosol generator. The inhalation unit is also contained in a secondary enclosure maintained at a negative pressure by an exhaust port in the ceiling to prevent any escape of aerosol into the room in case of malfunction. All effluent air from the inhalation system is filtered by a HEPA filter prior to release into the environment. The nose-only exposure system used in this manuscript was purchased from a single vendor (see supplemental Table of Materials).

\section{Protocol}

The mice used in these studies were cared for in accordance with the Guide for the Care and Use of Laboratory Animals, eighth edition ${ }^{24}$. Mice were group housed at an Association for Assessment and Accreditation of Laboratory Animal Care (AAALAC) international-accredited facility in sterile ventilated microisolator housing on corn cob bedding. When measuring bronchoconstriction, mice were anesthetized with $100 \mathrm{mg} / \mathrm{kg}$ i.p. pentobarbital and depth of anesthesia was monitored by lack of toe pinch reflex and maintained by i.p. anesthesia as necessary. At the end of the experiments, mice were euthanized by cervical dislocation after barbiturate overdose. Verification of euthanasia was confirmed by lack of respiration. No survival surgeries were performed on the mice. All research protocols were approved by the Institutional Animal Care and Use Committee (IACUC).

\section{Formulation and Device Selection for Generation of Pharmaceutical Aerosols}

NOTE: Formulation and device selection are dependent on the physicochemical properties of the individual drug to be aerosolized, therefore general protocols are presented below and the reader is referred to the reviews by Zeng ${ }^{25}$ and O'Riordan ${ }^{26}$.

\section{Dry powder aerosols}

1. Micronize drug dry powders in a ball mill, jet mill or similar device ${ }^{27}$ and ensure the micronized particle size distribution (PSD) contains particles of respirable (0.5-5 $\mu \mathrm{m}$ mass median aerodynamic diameter, MMAD) particle size. Blend potent test compounds that require dilution with micronized lactose.

NOTE: If there is not enough micronized powder to determine the PSD with a cascade impactor, the PSD of a small (sub-milligram) sample of the micronized powder can be measured by light scattering to confirm it contains small/respirable particles.

2. Generate the dry powder aerosol using a Wright dust feed dry powder aerosol generator. Pack the micronized drug/lactose powder into the cylindrical reservoir using a manual hydraulic press at approximately 1000 pounds per square inch (psi) to produce compacted cakes of powder used as input by the Wright dust feed aerosol generator ${ }^{17}$.

3. Screw the cylindrical reservoir onto the Wright dust feed advancing the reservoir until the scraper blade is in contact with the drug cake.

4. Connect the outlet of the Wright dust feed to a cyclone and the inlet to a compressed air source set to $15 \mathrm{~L} / \mathrm{min}$ airflow rate (maximum pressure $90 \mathrm{psi}$ ).

5. Set the feed rate control to 0.7 revolution per minute (rpm) and turn on the Wright dust feed aerosol generator. NOTE: $0.7 \mathrm{rpm}$ corresponds to a test article cake feed rate of $1 \mathrm{~g} / \mathrm{h}$ when using the small Wright dust feed cylindrical reservoir. The Wright dust feed scrapes a thin layer of compacted powder off the test article cake by rotating the reservoir. Air carries the dust out of the Wright dust feed, through a sonic nozzle for de-agglomeration, and into a cyclone to remove non-respirable particles and agglomerates.

6. Connect the outlet of the cyclone to the central aerosol plenum of the inhalation unit (Figure 1). 
NOTE: Compounds can be compressed from 300 to 1500 psi in the reservoir of the Wright dust feed. The object is to compress the particles enough so they will be retained in the reservoir when inverted, but not so much that the Wright dust feed cannot scrape off a thin layer for re-aerosolization. It must be remembered that the gauge on the manual hydraulic press reads in pounds and the plunger on the small dust feed reservoir has a surface area of about 0.25 square-inch. Therefore, 250 pounds of compression force on 0.25 square inch is equivalent to 1000 psi.

\section{Nebulized liquid aerosols}

1. Dissolve the drug in $100 \mathrm{~mL}$ of water or physiologic saline.

2. Load a $100 \mathrm{~mL}$ syringe with the drug solution and place the syringe into the syringe pump with a flow rate set at $1 \mathrm{~mL} / \mathrm{min}$.

3. Connect the syringe pump to the jet nebulizer and purge the air from the feed line leading to the nebulizer.

4. Connect the pressurized air source to the jet nebulizer and set the air flow meter to $10 \mathrm{~L} / \mathrm{min}$.

5. Insert the jet nebulizer into the pre-separator. The pre-separator connects the nebulizer to the central aerosol plenum of the inhalation unit (Figure 1).

NOTE: Many drug compounds have limited aqueous solubility and are better formulated as dry powder aerosols. If a stable suspension can be made from micronized (MMAD $<5 \mu \mathrm{M}$ ) compound, it may be used with the jet nebulizer. Caution should be used as the suspension may clog the nebulizer. Nebulizer feed concentrations from $1 \mathrm{mg} / \mathrm{mL}$ for potent compounds (like the bronchodilator ipratropium) to $40 \mathrm{mg} / \mathrm{mL}$ suspensions (for less potent compounds like salbutamol sulfate) have been used. The syringe pump feed rate is set at $1 \mathrm{~mL} / \mathrm{min}$ for a practical reason; to allow the aerosol concentration equilibration period and 45-min-long exposure to be completed without the need to reload the syringe.

\section{Aerosol Exposure Experiment Setup}

1. Measure the drug concentration (by collecting aerosol on an absolute filter) and particle size distribution (by collecting aerosol using a cascade impactor) of the aerosol that enters the inhalation unit post pre-separator/cyclone. Use these parameters along with the animal's minute ventilation, body weight, and exposure time to estimate the dose of drug deposited in the lungs.

1. Weigh the absolute filter and record the filter weight. Place the filter into the filter holder and assemble the filter holder. Connect the inlet of the absolute filter holder to a central aerosol plenum sample port and the outlet to a vacuum source set to sample the aerosol at a flow rate of $1 \mathrm{~L} / \mathrm{min}$ for the duration of the experiment.

NOTE: The mass of drug on the filter after sampling for $45 \mathrm{~min}$ can be in the sub-microgram range and/or be mixed with lactose, $\mathrm{NaCl}$ salt, or other vehicle. A microbalance that reads to 0.1 microgram is necessary. To get an accurate weight of drug deposited on the filter, the filter must be equilibrated and weighed in a humidity controlled environment. The weight of drug on the filters can only be used in dose calculation if there is no vehicle in the formulation or the vehicle is water. When there is a vehicle in the formulation other than water, the weight of substance on the filter only gives an estimated starting point for further analysis of drug content by high performance liquid chromatography (HPLC).

2. Weigh and record the weight of the 7 cascade impactor stage filters and one final "tail piece" filter. Place a filter on each of the seven stages of the cascade impactor and assemble the Mercer cascade impactor ${ }^{28}$. Connect the inlet of the cascade impactor to a central aerosol plenum sample port and the outlet to a vacuum source set to sample the aerosol at the flow rate the cascade impactor is calibrated at (usually 0.5 or $1 \mathrm{~L} / \mathrm{min}$ ) for the duration of the experiment.

3. Monitor the aerosol content of the inhalation unit with the real time aerosol monitor (see Table of Materials and Reagents) to confirm the aerosol generators are functional and producing a stable aerosol throughout the experiment. Connect the inlet of the real time aerosol monitor to a central aerosol plenum sample port and the outlet to a vacuum source set to sample the aerosol at a flow rate of 1 $\mathrm{L} / \mathrm{min}$ for the duration of the experiment.

NOTE: The signal from the real time aerosol monitor is reported in $\mu \mathrm{g} / \mathrm{L}$, but is calibrated for road dust and must be recalibrated for each individual drug aerosol to yield correct aerosol concentration values. Calibration is not needed to use the monitor to confirm the presence or absence and temporal stability of aerosol concentration.

4. Set the process control parameters (air flow, vacuum, pressure, aerosol generator power) to the values required that depends on the number of animals to be connected to the inhalation unit. The inhalation unit and aerosol generators are continuously controlled/ monitored by a computerized process control/data acquisition system (DACO) supplied by the manufacturer (see Table of Materials and Reagents). Air flow rate into the inhalation unit should be minimally approximately 2 times the total minute ventilation rate of all animals in the inhalation unit in order to avoid a buildup of $\mathrm{CO}_{2}$.

2. Load mice into nose-only restrainers before exposing to aerosol in the nose-only inhalation unit. Also load restraint stress control animals into restrainers to breathe room air.

NOTE: A dose/response experiment consists of multiple groups of mice exposed to aerosol for different amounts of time. Exposure time is used to control the dose each group receives during a dose/response experiment.

1. Angle the restraining tube up towards the ceiling while trying to load the animals, as they tend to run upward trying to escape. Pointing the tubes down while loading will encourage turning around and escaping out the back of the tube. Ensure the mouse's nose is oriented into the pointed end of the tube and fix the variable-position plunger into the back end of the restrainer.

NOTE: The plunger is fashioned to allow the tail of the mouse to protrude from the holder, which allows the mouse to regulate its body temperature while in the restrainer.

2. Adjust the plunger to allow the mice to rotate, but not turn head to tail; to ensure the aerosol is inhaled.

3. Continuously monitor the mice while in the restraining tubes. After positioning the plunger, small mice ( $<20$ grams $)$ often attempt to turn head to tail in the tubes and adopt a U-position where they have trouble breathing. This turning behavior is most prevalent in the first 5 minutes of restraint, after that the mice rarely attempt to turn head to tail. 


\section{Aerosol Delivery}

1. Insert stoppers to plug the delivery ports of the inhalation unit and activate the aerosol generator, compressed air flow controller, and inhalation unit vacuum pump from within the process control software.

2. Once the readings from the real time aerosol monitor demonstrate the aerosol concentration has to come to equilibrium ( 30 min, Figure 2), begin removing stoppers and inserting the nose-only restraining tubes containing mice into the inhalation unit. Repeat until all mice to be exposed to drug are connected to the inhalation unit.

NOTE: In the example experiment, the total air flow supplied to the inhalation unit via the aerosol generator and dilution air is set to supply a $0.5 \mathrm{~L} / \mathrm{min}$ flow rate to each of animal exposure ports in use. For example, a $15 \mathrm{~L} / \mathrm{min}$ total air flow is enough to supply each of the 30 ports in the inhalation unit. This is much more air flow than required by the minute ventilation of the mice, but a larger air flow is necessary to supply the energy (pressure drop across the aerosol generator) to produce (atomize/de-agglomerate) the aerosol.

3. Once all animals are loaded into the exposure unit, turn on the vacuum sampling pumps that are connected to the absolute filter and cascade impactor using the process control software.

4. When all exposures are completed, turn off the aerosol generator and remove remaining mice from inhalation unit. NOTE: Animals will be handled in a change station hood or by personnel wearing a facemask. After conclusion of aerosol delivery, the mice are removed from the tubes and the tubes are sanitized after each use.

\section{Calculation of the Deposited Dose}

1. To calculate the deposited dose ${ }^{29}$ in $\mu \mathrm{g} / \mathrm{kg}$ (Equation 1) multiply the concentration of the drug in the aerosol ( $\left.\mu \mathrm{g} / \mathrm{L}\right) \times \mathrm{minute}$ ventilation (L/min) $x$ duration of exposure $(\mathrm{min}) \mathrm{x}$ inhalable fraction $\mathrm{x}$ pulmonary deposition fraction and divide by the body weight $(\mathrm{kg})$.

2. Calculate the aerosol concentration $(\mu \mathrm{g} / \mathrm{L})$ by dividing the mass of drug on the absolute filter $(\mu \mathrm{g})$ by the air flow rate through the filter ( $\mathrm{L} /$ $\mathrm{min}$ ) multiplied by the sampling time ( $\mathrm{min})$. Estimate the mouse's minute ventilation by an allometric equation based on body weight ${ }^{30}$. The inhalable fraction is 1 as the aerosol was passed through a pre-separator to remove non-respirable particles, and the pulmonary deposition fraction is determined from the drug aerosol MMAD (Figure 3) using experimental calibration curves from monodisperse aerosols (Figure $4)^{31}$.

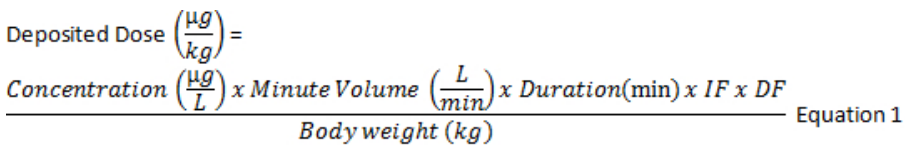

\section{Representative Results}

The bronchodilator ipratropium bromide was dissolved in $0.9 \%$ normal saline at a concentration of $1 \mathrm{mg} / \mathrm{mL}$. A $100 \mathrm{~mL}$ syringe was filled with the ipratropium formulation solution and the syringe was inserted into a syringe pump set to feed the jet nebulizer (Figure 1) at a flow rate of $1 \mathrm{~mL} / \mathrm{min}$. Activating the control system for the inhalation unit initiates a $10 \mathrm{~L} / \mathrm{min}$ air flow to the jet nebulizer, $5 \mathrm{~L} / \mathrm{min}$ dilution air flow to the dilution chamber, and $15.5 \mathrm{~L} / \mathrm{min}$ of vacuum air flow to draw the drug aerosol out of the inhalation unit once it passes the nose of the mice. The syringe pump and real time aerosol monitor sample pump were activated. Twenty-four mice were inserted into the inhalation unit after the real time aerosol monitor confirms the aerosol concentration in the inhalation unit had come to equilibrium (15-30 min, Figure 2). The sample pumps for the absolute filter $(1 \mathrm{~L} / \mathrm{min})$ and cascade impactor $(0.5 \mathrm{~L} / \mathrm{min})$ were turned on once all mice were connected to the inhalation unit. The first group of 8 mice was removed from the inhalation unit after $5 \mathrm{~min}$, the second group after $15 \mathrm{~min}$, and the third group after 45 min. Bronchoconstriction ${ }^{32}$ was measured as the increase in respiratory system resistance (Rrs) to a single nebulized dose of methacholine $(30 \mathrm{mg} / \mathrm{mL})$ delivered $2 \mathrm{~h}$ after dosing ipratropium using the rodent respirator ${ }^{33}$. The percent increase in Rrs for each animal, calculated as the maximum Rrs over the 3-min interval after nebulized methacholine (Rmax) minus the Rrs value of the baseline measurement before methacholine (Rbase) divided by Rbase (\% increase in Rrs = (Rmax-Rbase)/Rbase), was used to quantitate the bronchoconstriction.

The aerosol deposited on the cascade impactor filters and absolute filter during the $45 \mathrm{~min}$ of aerosol exposure was dissolved in $50 \%$ acetonitrile and the mass of ipratropium quantitated by HPLC (Table 1). The MMAD x/ GSD of the ipratropium aerosol was calculated to be $1.7 \times / 1.5 \mu \mathrm{m}$ (Figure 3) and a deposition fraction (DF) of 0.037 for mice was used for an aerosol with an MMAD of $1.7 \mu$ m (Figure 4). The inhalable fraction (IF) was 1 as the aerosol was passed through a pre-separator to remove non-respirable particles. The average concentration of ipratropium in the aerosol $(0.5 \mu \mathrm{g} / \mathrm{L})$ was calculated from mass of ipratropium on the absolute filter $(22 \mu \mathrm{g})$ divided by the flow rate of air pulled through the filter $(1 \mathrm{~L} / \mathrm{min})$ multiplied by the sampling time $(45 \mathrm{~min})$. The average body weight of the mice was $0.019 \mathrm{~kg}$ and their predicted minute ventilation was calculated as $0.021 \mathrm{~L} / \mathrm{min}$. Using Equation 1, the deposited doses for the 5,15 , and 45 min exposure groups were $0.1,0.3$, and $0.9 \mu \mathrm{g} / \mathrm{kg}$; respectively.

Ipratropium inhibited nebulized methacholine induced bronchoconstriction in 8 week old C57BI/6 mice. Methacholine increased Rrs within seconds of nebulized administration (Figure 5, top panel). The Rrs of the control group (exposed to room air instead of ipratropium aerosol) increased from a baseline value of $0.62 \pm 0.03 \mathrm{cmH}_{2} \mathrm{O}^{*} \mathrm{~s} / \mathrm{mL}$ to a maximum value of $1.66 \pm 0.12 \mathrm{~cm} \mathrm{H}_{2} \mathrm{O}^{*} \mathrm{~s} / \mathrm{mL}$, representing a $168 \pm 9 \%$ increase in Rrs, $70 \mathrm{~s}$ after methacholine aerosol administration. The percent increase in respiratory system resistance was inhibited in a dose dependent manner by inhaled doses of ipratropium. The percent increase in Rrs was inhibited by $51 \pm 9 \%, 79 \pm 14 \%$, and $89 \pm 2 \%$ (Figure 5, bottom panel) at inhaled deposited doses of ipratropium of $0.1,0.3$, and $0.9 \mu \mathrm{g} / \mathrm{kg}$, respectively. 


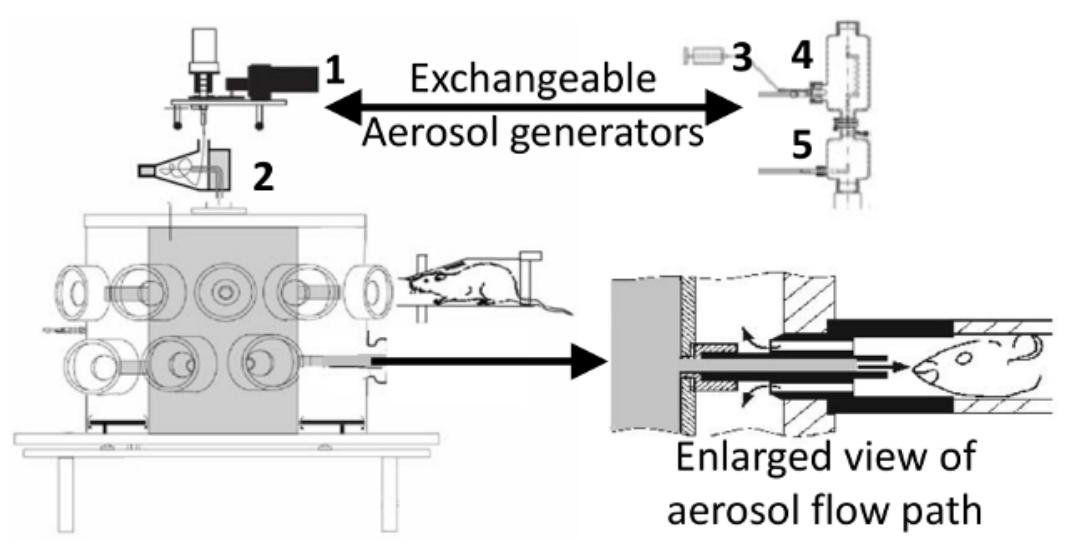

Figure 1: Inhalation unit with dry powder aerosol generator attached. The nebulizer used to generate aerosol from liquid formulations is shown on the right. 1) Wright dust feed, 2) cyclone, 3) syringe pump, 4) jet nebulizer inserted into pre-separator, 5) dilution air mixer. When delivering nebulized aerosols, the dust feed and cyclone are replaced by the syringe pump, jet nebulizer/pre-separator, and dilution air mixer. The enlarged view (modified from Oldham ${ }^{21}$ ) shows the aerosol flow path at the breathing zone around animal's nose that is created by inserting the restraining tube into the inhalation unit. The aerosol fills the central aerosol (gray) plenum of the inhalation unit, flows out to the animal's nose where it is pulled back by a slight vacuum in the outer (white) plenum and into the waste collection filters (not shown). Please click here to view a larger version of this figure.

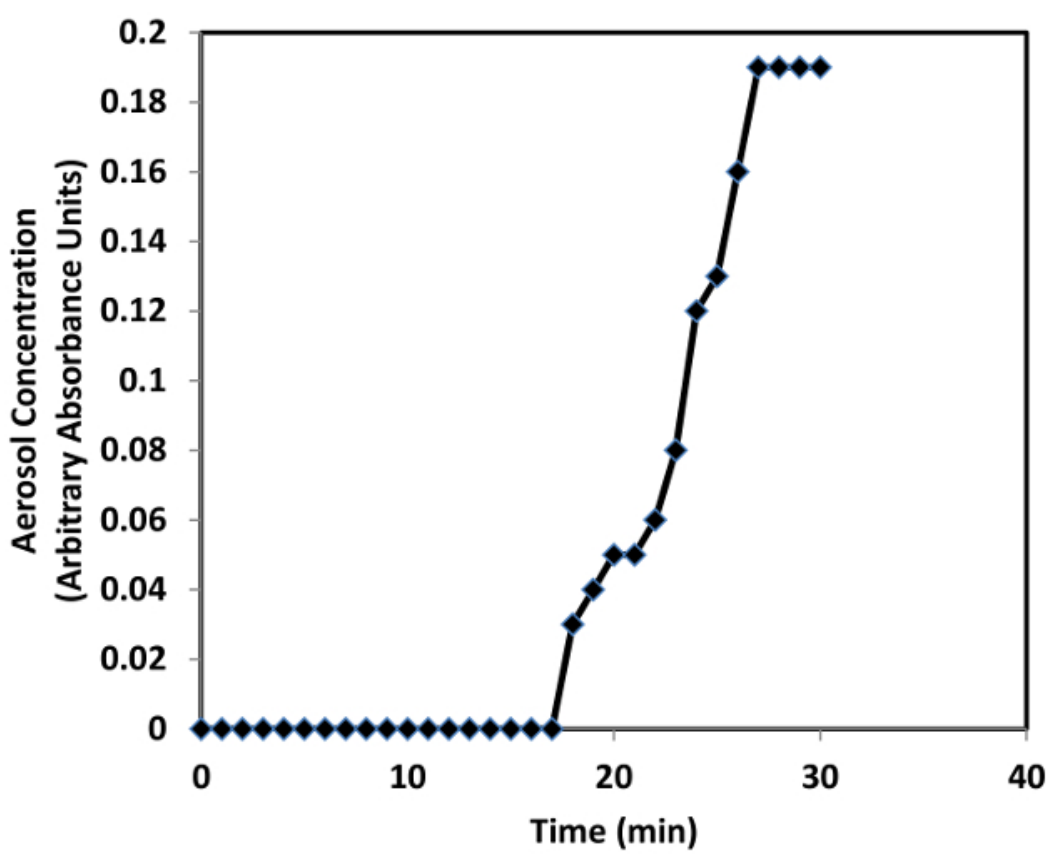

Figure 2: Real time aerosol monitor measurements confirm the aerosol concentration in the inhalation unit reaches equilibrium $~ 30$ min after turning on the aerosol generator. Please click here to view a larger version of this figure. 


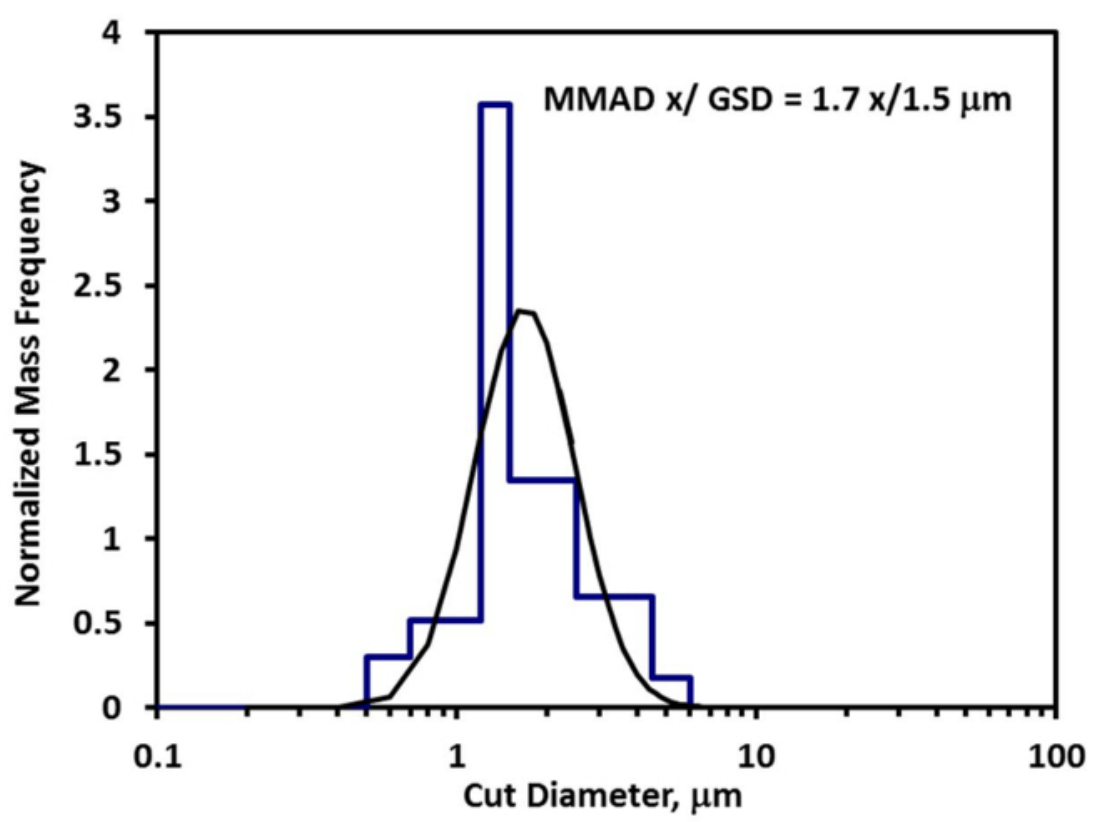

Figure 3: Ipratropium mass collected on each stage of the cascade impactor (blue bar graph) overlaid with curve fit (black curve) used to calculate the MMAD and GSD of the aerosol particle size distribution. Please click here to view a larger version of this figure.

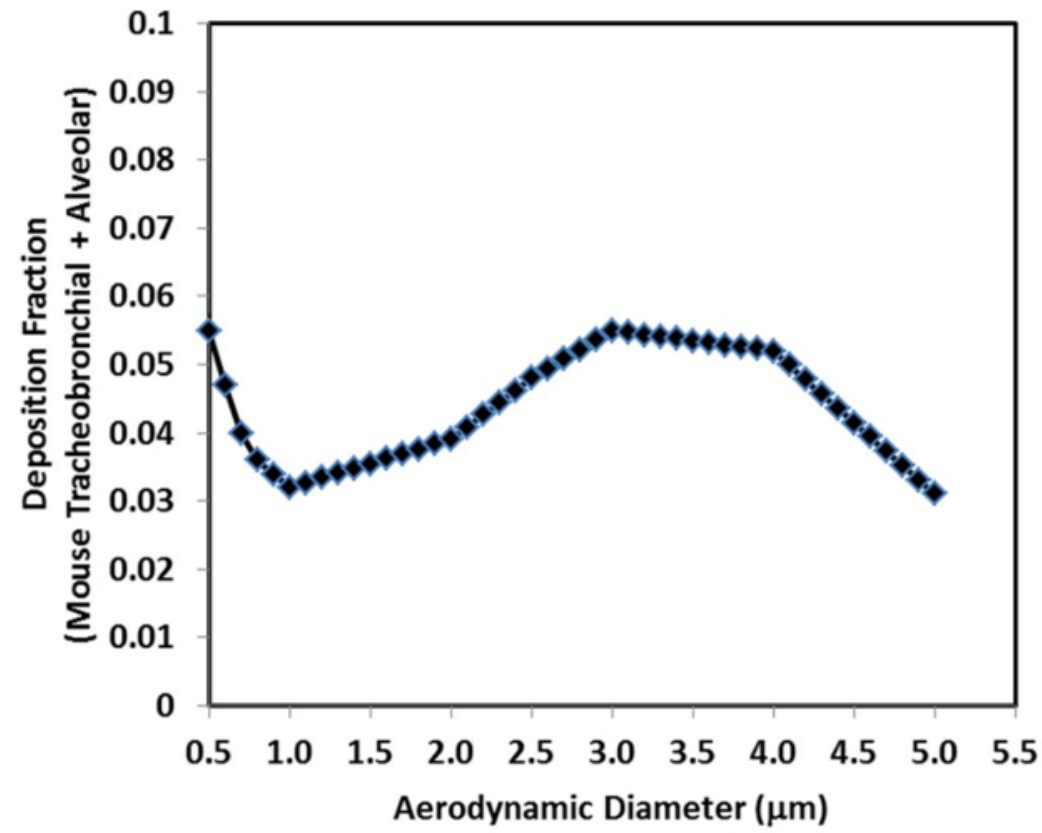

Figure 4: Deposition fraction for mouse lungs as a function of MMAD for aerosols delivered by nose-only inhalation (as modified from Hsieh $^{31}$ ). For particle sizes less than $0.5 \mu \mathrm{m}$ most of the aerosol is exhaled and does not deposit in the lungs (like cigarette smoke). For particle sizes greater than $5 \mu \mathrm{m}$, most of the aerosol is filtered out by the nose. Please click here to view a larger version of this figure. 

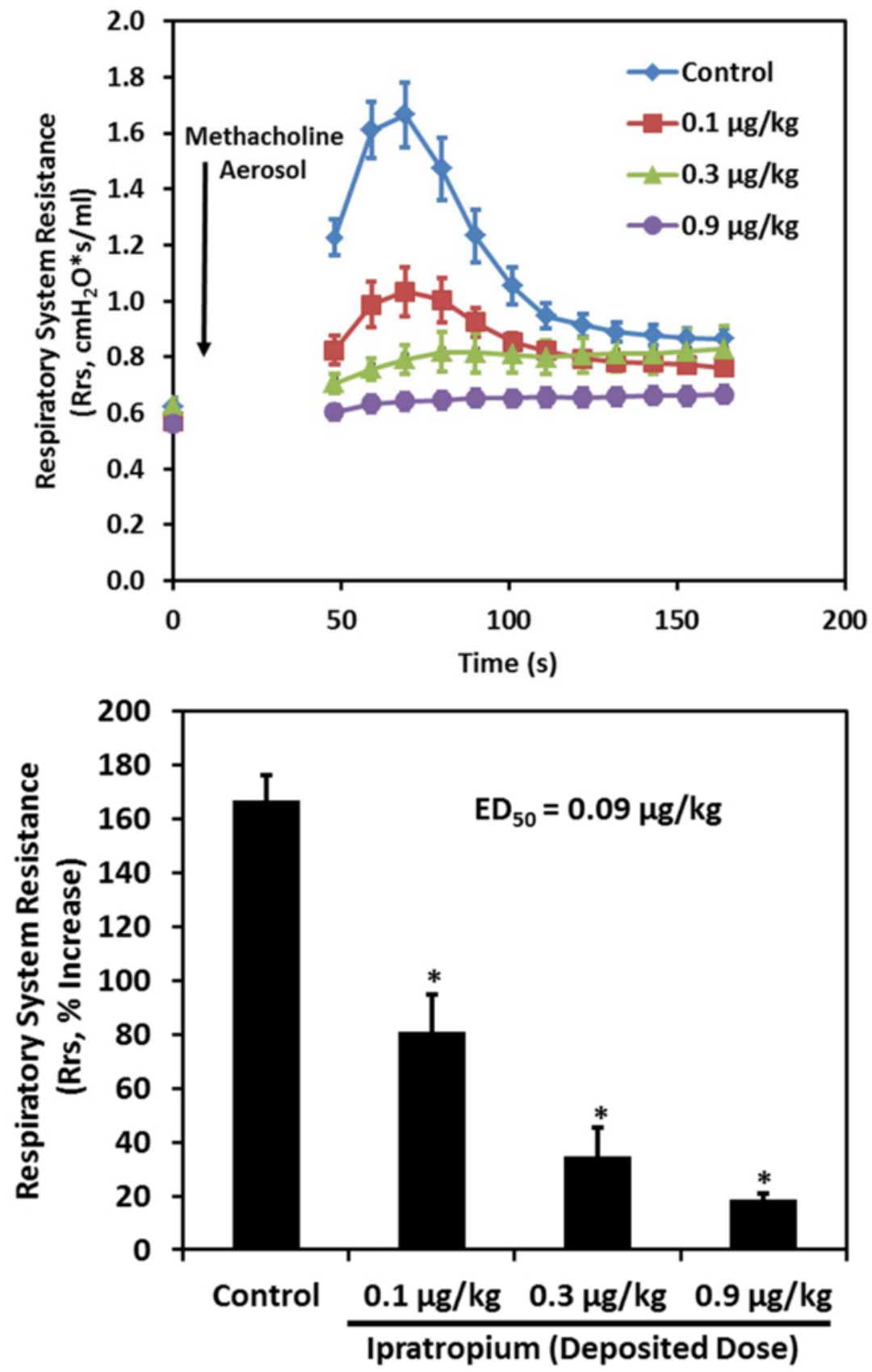

Figure 5: The rapid increase in respiratory system resistance induced by nebulized methacholine administered to mice is blocked by ipratropium delivered by nose-only inhalation to mice $(\mathbf{n}=\mathbf{8}$ per group, top panel). Ipratropium inhibited the methacholine induced increase in respiratory system resistance with an $\mathrm{ED}_{50}$ of $0.1 \mu \mathrm{g} / \mathrm{kg}$ deposited dose ( ${ }^{*} \mathrm{p}<0.05 \mathrm{vs}$. control, bottom panel). Please click here to view a larger version of this figure. 


\begin{tabular}{|l|l|l|}
\hline Cascade impactor data used to calculate MMAD and GSD \\
\hline Stage Filter & $\begin{array}{l}\text { lpratropium on filter } \\
\text { by HPLC }(\mathrm{mg})\end{array}$ & $\begin{array}{l}\text { Stage Cut } \\
\text { Diameter (mm) }\end{array}$ \\
\hline 1 & 0.002 & 6 \\
\hline 2 & 0.19 & 4.5 \\
\hline 3 & 1.43 & 2.5 \\
\hline 4 & 2.55 & 1.5 \\
\hline 5 & 2.95 & 1.2 \\
\hline 6 & 1.03 & 0.7 \\
\hline 7 & 0.37 & 0.5 \\
\hline
\end{tabular}

Table 1: Cascade impactor data used to calculate MMAD and GSD

\section{Discussion}

A nose-only inhalation system and its operation to deliver pharmaceutical aerosols to the lungs of rodents has been described. Restraining animals in nose-only holders is a commonly used method for exposing animals to airborne material. An experiment was conducted demonstrating the anticholinergic bronchodilator ipratropium bromide ${ }^{34}$ can potently reverse methacholine induced bronchoconstriction when delivered by nose-only inhalation to mice with an $\mathrm{ED}_{50}$ of $0.1 \mu \mathrm{g} / \mathrm{kg}$ deposited dose. A greater than 10 -fold increase in effective dose of ipratropium was required for bronchodilation following intratracheal delivery $\left(E D_{50}\right.$ intratracheal $=1.3 \mu \mathrm{g} / \mathrm{kg}$, data not shown) delivery. This is due to the non-homogeneous deposition pattern of drug in the lungs produced by intratracheal dosing ${ }^{3,5,10}$. 10-fold and greater effective dose differentials between inhaled and intratracheal dosing techniques for other drugs have been observed previously by others ${ }^{4}$.

The non-homogeneous deposition pattern of drug in the lungs produced by intratracheal dosing also slows drug absorption by the lung ${ }^{35}$, decreasing the rate at which the drug enters the systemic circulation and decreasing the chances of seeing systemic side effects. Therefore, to optimize the safety/efficacy (therapeutic index) ${ }^{36}$ of novel inhaled drugs, nose-only inhalation delivery should be used. Intratracheal deposition will give an inaccurate estimate of the TI by generating erroneously high effective doses in the lung and low doses to the systemic circulation.

As new drugs delivered by the inhaled route are developed, it is essential to appropriately translate the efficacious drug dose from preclinical efficacy studies to predict an efficacious human dose for clinical trials. The infamous death of Tusko the elephant ${ }^{37}$ is commonly cited in the literature to remind us to use allometric scaling to predict interspecies drug doses and that interspecies drug doses should not be linearly extrapolated on the basis of a simple comparison of body masses. It is common to use an allometric approach with an allometric exponent $\mathrm{b}$ of 0.67 to predict human drug doses from preclinical efficacy studies ${ }^{38}$. Using the mouse inhaled $\mathrm{ED}_{90}$ of $0.9 \mu \mathrm{g} / \mathrm{kg}$ for ipratropium, an allometric exponent of 0.67 , a mouse body mass of $0.03 \mathrm{~kg}$, and a human body mass of $60 \mathrm{~kg}$; an estimated human $E D_{90}$ of $0.07 \mu \mathrm{g} / \mathrm{kg}$ $\left(\left(0.9^{*}(0.03 / 60)^{(1-0.67)}=0.07\right)\right.$ can be calculated as the predicted human deposited dose from our mouse data. This predicted value is comparable to the actual human efficacious deposited dose of $0.26 \mu \mathrm{g} / \mathrm{kg}$, which can be calculated from the delivered dose of $40 \mu \mathrm{g}^{39}$ divided by the human body mass of $60 \mathrm{~kg}$ multiplied by a human oral inhalation pulmonary deposition fraction ${ }^{16}$ of $0.4\left((40 / 60)^{\star} 0.4=0.26\right)$. An estimate of the effective human inhaled deposited dose also helps to plan the delivered doses used in inhalation toxicology studies required for clinical trials ${ }^{40}$.

The specialized equipment and large amount of test compound (gram quantities) required for nose-only inhaled drug delivery can be significant limitations when developing the nose-only inhalation technique. A calibration run without animals present is necessary if a specific dose is required for a (usually toxicology and not an efficacy dose/response) study, and this calibration run will require more drug to be available. This calibration run is necessary because the physicochemical properties of each drug/formulation can vary enough to greatly affect the deposited dose of drug in the lungs. Optimization of the air and drug feed rates to the aerosol generator during the calibration run is required to achieve an aerosol particle size and concentration for the specific dose required. While the aerosol generator air and drug feed rates suggested in the methods are a reasonable starting point, there is potential that for a specific drug formulation a non-respirable (particle size $>5 \mathrm{~mm}$ ) aerosol will be generated. At the preclinical stage, there is often not enough drug available to do a calibration run and it is impossible to know a priori what doses (if any) are getting into the lungs. Also, static electricity is produced during the aerosol generation process and can influence the time required for the aerosol concentration to equilibrate. It is important to properly ground the equipment to minimize static charge. Another option to minimize static electricity is to add humidity to the air supplied to the inhalation unit, to increase conductivity and dissipate electrostatic charges on particles ${ }^{25}$. Humidifying the air supplied to the aerosol generator is not required for animal comfort during short $(<1 \mathrm{~h})$ dosing sessions but should be considered if longer dosing times are used.

Drugs can be delivered to the lungs of animals by passive nose-only inhalation or direct intratracheal administration methods that bypass nasopharyngeal deposition. Nose-only inhalation delivery is commonly used in the field of inhalation toxicology ${ }^{41}$ but is sparingly used early in the drug discovery process. A multidisciplinary research team is needed for conducting nose-only inhalation studies due to the: need for large quantities of drug, specialized knowledge required to formulate, generate, and characterize aerosols; operate complex equipment, and measure drug efficacy in animal models of respiratory disease. The delivery techniques described herein are used to develop small molecule inhaled drugs but in the future can be applied to develop inhaled biologics ${ }^{42,43}$. Hopefully the procedures and tips documented in this manuscript will facilitate new pre-clinical drug discovery and toxicology researchers to acquire the skills necessary to deliver drugs to rodents by aerosol inhalation. 


\section{Disclosures}

All authors employed by Amgen. Publication fees for this this video-article are paid by Amgen.

\section{Acknowledgements}

We acknowledge: Dr. Thomas Budiman at TSE Systems GmbH for his technical expertise and equipment customization. John Fry (Battelle Inc.) and Dr. Rudy Jaeger (CH Technologies Inc.) for their helpful discussions. Tian Wu, Sam Mboggo, April Miller, and Sean Davis (Amgen) for help with the experiments.

\section{References}

1. Chapman, R. W. et al. Pharmacology of a potent and selective inhibitor of PDE4 for inhaled administration. Eur J Pharmacol. 643 (2-3), 274-281 (2010).

2. Phillips, J. E. et al. Btk inhibitor RN983 delivered by dry powder nose-only aerosol inhalation inhibits bronchoconstriction and pulmonary inflammation in the ovalbumin allergic mouse model of asthma. J Aerosol Med Pulm Drug Deliv. 29 (3), 233-241 (2016).

3. Zecchi, R. et al. Impact of drug administration route on drug delivery and distribution into the lung: an imaging mass spectrometry approach. Eur J Mass Spectrom. 19 (6), 475-482 (2013).

4. Cooper, A. E., Ferguson, D., \& Grime, K. Optimisation of DMPK by the inhaled route: challenges and approaches. Curr Drug Metab. 13 (4), 457-473 (2012).

5. Leong, B. K., Coombs, J. K., Sabaitis, C. P., Rop, D. A., \& Aaron, C. S. Quantitative morphometric analysis of pulmonary deposition of aerosol particles inhaled via intratracheal nebulization, intratracheal instillation or nose-only inhalation in rats. J Appl Toxicol. 18 (2), 149-160 (1998).

6. Pauluhn, J. Overview of inhalation exposure techniques: strengths and weaknesses. Exp Toxicol Pathol. 57 Suppl $1111-128$ (2005).

7. Wong, B. A. Inhalation exposure systems: design, methods and operation. Toxicol Pathol. 35 (1), 3-14 (2007).

8. Phalen, R. F. Inhalation Studies Foundations and Techniques. 2nd edn Informa Healthcare (2009).

9. Siddiqui, S. et al. Pulmonary eosinophilia correlates with allergen deposition to the lower respiratory tract in a mouse model of asthma. Clin Exp Allergy. 38 (8), 1381-1390 (2008).

10. Brain, J. D., Knudson, D. E., Sorokin, S. P., \& Davis, M. A. Pulmonary distribution of particles given by intratracheal instillation or by aerosol inhalation. Environ Res. 11 (1), 13-33 (1976).

11. Liu, F., Li, W., Pauluhn, J., Trubel, H., \& Wang, C. Lipopolysaccharide-induced acute lung injury in rats: comparative assessment of intratracheal instillation and aerosol inhalation. Toxicology. 304 158-166 (2013).

12. Bivas-Benita, M., Zwier, R., Junginger, H. E., \& Borchard, G. Non-invasive pulmonary aerosol delivery in mice by the endotracheal route. Eur J Pharm Biopharm. 61 (3), 214-218 (2005).

13. Morello, M. et al. Dry-powder pulmonary insufflation in the mouse for application to vaccine or drug studies. Tuberculosis (Edinb). 89 (5), 371-377 (2009).

14. Guillon, A. et al. Pulmonary delivery of dry powders to rats: tolerability limits of an intra-tracheal administration model. Int J Pharm. 434 (1-2), 481-487 (2012).

15. Pauluhn, J., \& Mohr, U. Inhalation studies in laboratory animals--current concepts and alternatives. Toxicol Pathol. 28 (5), $734-753$ (2000).

16. Snipes, M. B., McClellan, R. O., Mauderly, J. L., \& Wolff, R. K. Retention patterns for inhaled particles in the lung: Comparisons between laboratory animals and humans for chronic exposures. Health Phys. 57 (Sup 1) 69-78 (1989).

17. Wright, B. M. A new dust-feed mechanism. J Sci Inst. 27 12-15 (1950).

18. Scheuch, G., \& Siekmeier, R. Novel approaches to enhance pulmonary delivery of proteins and peptides. J Physiol Pharmacol. $\mathbf{5 8} \mathbf{S u p p l} \mathbf{5}$ (Pt 2), 615-625 (2007).

19. Phillips, J. E., Ji, L., Rivelli, M. A., Chapman, R. W., \& Corboz, M. R. Three-dimensional analysis of rodent paranasal sinus cavities from X-ray computed tomography (CT) scans. Can J Vet Res. 73 (3), 205-211 (2009).

20. Wolff, R. K. Toxicology studies for inhaled and nasal delivery. Mol Pharm. (2015).

21. Oldham, M. J., Phalen, R. F., \& Budiman, T. Comparison of predicted and experimentally measured aerosol deposition efficiency in BALB/C mice in a new nose-only exposure system. Aerosol Sci Technol. 43 (10), 970-977 (2009).

22. Cannon, W. C., Blanton, E. F., \& McDonald, K. E. The flow-past chamber: an improved nose-only exposure system for rodents. Am Ind Hyg Assoc J. 44 (12), 923-928 (1983).

23. Narciso, S. P., Nadziejko, E., Chen, L. C., Gordon, T., \& Nadziejko, C. Adaptation to stress induced by restraining rats and mice in nose-only inhalation holders. Inhal Toxicol. 15 (11), 1133-1143 (2003).

24. Guide for the Care and Use of Laboratory Animals. 8th edn National Academies Press (2011).

25. Zeng, X., Martin, G., \& Marriott, C. Particulate Interactions in Dry Powder Formulations for Inhalation. Taylor \& Francis (2001).

26. O'Riordan, T. G. Formulations and nebulizer performance. Respir Care. 47 (11), 1305-1313 (2002).

27. Pilcer, G., \& Amighi, K. Formulation strategy and use of excipients in pulmonary drug delivery. Int J Pharm. 392 (1-2), 1-19 (2010).

28. Mercer, T. T., Tillery, M. I., \& Newton, G. J. A multi-stage, low flow rate cascade impactor. J. Aerosol Sci. 1 (1), 9-15 (1970).

29. Forbes, B. et al. Challenges in inhaled product development and opportunities for open innovation. Adv Drug Deliv Rev. 63 (1-2), $69-87$ (2011).

30. Alexander, D. J. et al. Association of inhalation toxicologists (AIT) working party recommendation for standard delivered dose calculation and expression in non-clinical aerosol inhalation toxicology studies with pharmaceuticals. Inhal Toxicol. 20 (13), 1179-1189 (2008).

31. Hsieh, T. H., Yu, C. P., \& Oberdorster, G. Deposition and clearance models of Ni compounds in the mouse lung and comparisons with the rat models. Aerosol Sci Technol. 31 (5), 358-372 (1999).

32. Phillips, J. E. et al. House dust mite models: will they translate clinically as a superior model of asthma? J Allergy Clin Immunol. 132 (1), 242-244 (2013). 
33. McGovern, T. K., Robichaud, A., Fereydoonzad, L., Schuessler, T. F., \& Martin, J. G. Evaluation of respiratory system mechanics in mice using the forced oscillation technique. JoVE. (75), e50172 (2013).

34. Storms, W. W., DoPico, G. A., \& Reed, C. E. Aerosol Sch 1000. An anticholinergic bronchodilator. Am Rev Respir Dis. 111 (4), $419-422$ (1975).

35. Schanker, L. S., Mitchell, E. W., \& Brown, R. A., Jr. Species comparison of drug absorption from the lung after aerosol inhalation or intratracheal injection. Drug Metab Dispos. 14 (1), 79-88 (1986).

36. Biju, P. et al. Steroidal C-21 mercapto derivatives as dissociated steroids: discovery of an inhaled dissociated steroid. Bioorg Med Chem Lett. 21 (21), 6343-6347 (2011).

37. West, L. J., Pierce, C. M., \& Thomas, W. D. Lysergic acid diethylamide: Its effects on a male asiatic elephant. Science. 138 (3545), 1100-1103 (1962).

38. Boxenbaum, H., \& DiLea, C. First-time-in-human dose selection: allometric thoughts and perspectives. J Clin Pharmacol. 35 (10), $957-966$ (1995).

39. Spina, D. Current and novel bronchodilators in respiratory disease. Curr Opin Pulm Med. 20 (1), 73-86 (2014).

40. Degeorge, J. J. et al. Considerations for toxicology studies of respiratory drug products. Regul.Toxicol.Pharmacol. 25 (2), $189-193$ (1997).

41. McClellan, R. O., \& Henderson, R. F. Concepts in Inhalation Toxicology. Taylor \& Francis Group (1989).

42. Patton, J. S. et al. The particle has landed--characterizing the fate of inhaled pharmaceuticals. J Aerosol Med Pulm Drug Deliv. 23 Suppl 2 S71-87 (2010).

43. Depreter, F., Pilcer, G., \& Amighi, K. Inhaled proteins: Challenges and perspectives. Int J Pharm. 447 (1-2), $251-280$ (2013). 